\title{
Validation study of accelerometer in measuring some sleep parameters in adults-reply
}

\author{
Paul H. Lee ${ }^{1}$
}

Received: 3 August 2017 / Accepted: 7 August 2017 / Published online: 14 August 2017

(C) Springer International Publishing AG 2017

Dr. Kawada raised three concerns about our study. First, the validity of the reference accelerometer, Actiwatch 2, was questioned, as there existed only validation study in children and adolescents (against polysomnography). Second, there are a number of thresholds for classifying sleep and awake epochs and they perform differently in measuring different sleep parameters. Third, subjects with insomnia may have little motion during wakefulness and these wakefulness time might be misclassified as sleep by accelerometers.

These concerns are all against the validity of accelerometers in measuring sleep parameters. While they are important considerations for studies using accelerometer-measured sleeping parameters, the conclusions of our study were not affected by these concerns. First, the sleeping profile of the Actiwatch 2 validation study Dr. Kawada was referring to, Meltzer et al. 2012 [1], was similar to our sample (wake after sleep onset 69.2 vs $73.7 \mathrm{~min}$, sleep efficiency 82.3 vs $86.1 \%$ ); therefore, the results of this Actiwatch 2 validation study should be able to extend to our sample. Second, we reported the agreement of Actiwatch 2 and Actigraph for high, medium, and low thresh- olds plus other four classification methods (Tables 3 and 4 in our paper), and researchers can decide which classification method to be used according to the agreements. Third, it is a fact that accelerometers measure the motion of the wrist as a proxy of sleep and other characteristics of the human body that can help classifying a subject's sleep/awake status, for example, brain motion and heart rate are not considered. However, empirical data showed that sleep parameters estimated with accelerometers had acceptable validity against the gold standard measurement (polysomnography). Thus, accelerometers are widely used to measure habitual sleep pattern and the third point Dr. Kawada raised should be a minor limitation only.

\section{Reference}

1. Meltzer LJ, Walsh CM, Traylor J, Westin AML (2012) Direct comparison of two new actigraphs and polysomnography in children and adolescents. Sleep 35(1):159-166
Paul H. Lee

paul.h.lee@polyu.edu.hk

1 School of Nursing, Hong Kong Polytechnic University, Hung Hom, Kowloon, Hong Kong 